\title{
Rapid Onset of Intranasal Esketamine in Patients with Treatment Resistant Depression and Major Depression with Suicide Ideation: A Meta-Analysis
}

\author{
Sheng-Min Wang ${ }^{1}$, Nak-Young Kim², Hae-Ran Na ${ }^{1}$, Hyun Kook Lim ${ }^{1}$, Young Sup Woo ${ }^{1}$, Chi-Un Pae', \\ Won-Myong Bahk ${ }^{1}$ \\ ${ }^{1}$ Department of Psychiatry, College of Medicine, The Catholic University of Korea, Seoul, ${ }^{2}$ Department of Psychiatry, Keyo Hospital, Uiwang, Korea
}

\begin{abstract}
Objective: We performed a meta-analysis of randomized double-blinded placebo controlled trials (DB-RCTs) to investigate efficacy and safety of intranasal esketamine in treating major depressive disorder (MDD) including treatment resistant depression (TRD) and major depression with suicide ideation (MDSI).

Methods: Mean change in total scores on Montgomery-Åsberg Depression Rating Scale (MADRS) from baseline to different time-points were our primary outcome measure. Secondary efficacy measures included rate of remission of depression and resolution of suicidality.

Results: Eight DB-RCTs (seven published and one un-published) covering 1,488 patients with MDD were included. Esketamine more significantly improved MADRS total scores than placebo starting from $2-4$ hours after the first administration (standardized mean difference, $-0.41[95 \% \mathrm{Cl},-0.58$ to -0.25$], p<0.00001$ ), and this superiority maintained until end of double-blinded period (28 days). Sub-group analysis showed that superior antidepressant effects of esketamine over placebo in TRD and MDSI was observed from 2-4 hours, which was maintained until 28 days. Resolution of suicide in MDSI was also greater for esketamine than for placebo at 2-4 hours (OR of 2.04, 95\% Cls, 1.37 to $3.05, p=0.0005)$, but two groups did not statistically differ at 24 hours and day 28 . Total adverse events (AEs), and other common AEs including dissociation, blood pressure increment, nausea, vertigo, dysgeusia, dizziness, and somnolence were more frequent in esketamine than in placebo group.

Conclusion: Esketamine showed rapid antidepressant effects in patients with MDD, including TRD and MDSI. The study also suggested that esketamine might be associated with rapid anti-suicidal effects for patients with MDSI.
\end{abstract}

KEY WORDS: Esketamine; Depression; Treatment resistant depression; Suicide; Meta-analysis.

\section{INTRODUCTION}

Major depressive disorder (MDD) is a common debilitating disease with a lifetime prevalence of $15-20 \%$, and it is known to cause severe functional impairment $[1,2]$. Multiple antidepressants are available, but approximately one-third of patients with MDD fail to achieve adequate response or remission and become treatment-resistant depression (TRD) [3]. Besides low remission and response

Received: February 1, 2021 / Revised: February 15, 2021

Accepted: February 17, 2021

Address for correspondence: Won-Myong Bahk

Department of Psychiatry, Yeouido St. Mary's Hospital, College of

Medicine, The Catholic University of Korea, 10 63-ro,

Yeongdeungpo-gu, Seoul 07345, Korea

E-mail: wmbahk@catholic.ac.kr

ORCID: https://orcid.org/0000-0002-0156-2510 rate, delayed onset of efficacy is another important limitation of conventional antidepressants [4,5]. Moreover, around $10-20 \%$ of patients with MDD attempt suicide over their lifetimee, and $3.4 \%$ of patients with MDD actually commit or complete suicide [6,7]. However, due to therapeutic lag between administration of antidepressant and onset of clinical improvement, patients having major depression with suicide ideation (MDSI) remain symptomatic and at risk of suicidal behavior and self-harm for more than two weeks [8]. The monoamine hypothesis of depression received criticisms for more than a decade, and studies suggested that patients with TRD may need novel antidepressants with different mechanisms of action $[9,10]$. Thus, additional antidepressant having novel mechanism of action, higher potency, faster onset of ac-

(ㄷ) This is an Open-Access article distributed under the terms of the Creative Commons Attribution Non-Commercial License (http://creativecommons.org/licenses/by-nc/4.0) which permits unrestricted non-commercial use, distribution, and reproduction in any medium, provided the original work is properly cited. 
tion, and anti-suicidal effect are urgently needed [11].

Esketamine is a nonselective, noncompetitive antagonist at the $N$-methyl-D-aspartate (NMDA) receptor which modulates glutamatergic transmission [12]. It is an S-enantiomer of ketamine, which is known to have a higher affinity for the NMDA receptor than the R-enantiomer [13]. The US Food and Drug Administration (FDA) approved intranasal esketamine in conjunction (augmentation) with an oral antidepressant first for the treatment of TRD in 2019 [14]. Three double-blinded randomized placebo controlled trials (DB-RCTs) have shown its anti-suicidal effect [15-17], so the US FDA further approved intranasal esketamine augmentation to treatment depressive symptoms in adults with MDD having suicidal ideation or behavior [18].

An earlier study of intranasal esketamine showed its rapid onset of action in TRD [19]. However, findings from subsequent DB-RCTs of intranasal esketamine have been mixed in terms of its rapid antidepressant effect $[20,21]$. Although studies confirmed its anti-suicidal effects in patients with MDSI, but whether or not the anti-suicidal effects are rapid is still obscure. In terms of understanding efficacy and safety of a new drug, meta-analysis is important because it can overcome limitation of small sample sizes, increase statistical power of group comparisons, enhance generalizability of DB-RCTs, and quantify inconsistencies of DB-RCTs $[22,23]$. An initial meta-analysis for intranasal esketamine showed that significant superiority of intranasal esketamine over placebo with regard to response and remission in patients with MDD were noted as early as two hours [24]. However, the study included only four DB-RCTs which precluded more detailed elucidation of publication bias for outcome measures. In addition, due to small sample size, the study was unable to confirm its effects in MDSI. A more recent study by Papakostas et al. [25] also showed that adjunctive intranasal esketamine was significantly more effective than placebo for Montgomery-Åsberg Depression Rating Scale (MADRS) score change, response, and remission. However, besides having small study numbers (5 DB-RCTs), the timing of primary outcome or end-point measurements differed depending on the studies, but the meta-analysis did not specify their efficacies according to different time after esketamine administration. The study also failed to address whether or not esketamine have rapid antidepressant effect.

We performed a meta-analysis and studied efficacy and safety of intranasal esketamine in treatment of patients with MDD. We also aimed to investigate its rapid antidepressive actions in patients with TRD and MDSI

\section{METHODS}

\section{Sources of Data}

Three investigators (SMW, NKK, and YSW) independently searched from December 1st, 2020 to January 10th, 2021 using following terms: "esketamine," and "depression" (Mesh) at PubMed, Embase, PubMed, PsycINFO, Cumulative Index to Nursing and Allied Health Literature (CINAHL), and Web of Science for published articles. No restrictions were utilized for publication date. In terms of clinical trials, Cochrane Central Register of Controlled Trials Library and ClinicalTrials.gov (www.clinicaltrials.gov) was explored. We also manually searched reference lists from identified articles and reviews to find additional studies. Two other authors (HRN and HKL) re-evaluated potentially eligible papers to determine whether they truly met the selection criteria. The last two authors (CUP and WMB) discussed and reached a consensus for disagreements.

\section{Study Criteria and Data Extraction}

Primary inclusion criteria were all DB-RCTs comparing adjunctive treatment of intranasal esketamine with standard antidepressants for MDD. To be included in our meta-analysis, studies were required to: 1 ) have placebo as a comparator, regardless of having an active comparator, 2) exclusively focused on patients with MDD 3) have clearly described all inclusion and exclusion criteria. No restrictions were utilized for severity of MDD, gender, treatment basis (i.e., inpatient or outpatient), dose range, or study location. Three investigators (SMW, NKK, and YSW) who conducted initial data search also extracted the data. In addition, if a DB-RCT contained multiple double-blinded phases (i.e., Daly et al. [19]), only data from the first period were extracted and analyzed. We also assessed quality of DB-RCTs based on recommendations of Cochrane Review [26].

\section{Study Outcomes}

The primary outcome measures were change of MADRS total score from baseline to different time points until the end of double blinded phase. The secondary efficacy measures were rate of study-defined remission and reso- 
lution of suicidality at different time points during the double blinded phase. In terms of safety and tolerability, total number of adverse events (AEs) and common AEs including dissociation, blood pressure increment, nausea, vertigo, dysgeusia, dizziness, somnolence, and headache were included in the meta-analysis.

\section{Statistical Analysis}

Review Manager version 5.4 software (Cochrane Collaboration, Oxford, UK) was used to undertake statistical analysis. Standardized mean difference (SMD) using method developed by Hedges (Hedges g) with 95\% confidence intervals (95\% Cls) and odds ratio (OR) with 95\% Cls using Mantel-Haenszel method were used for continuous and dichotomous outcome measures respec- tively. Cohen's classification can be used to assess effect size: small $=\mathrm{SMD}<0.2$, medium $=\mathrm{SMD}$ of 0.5 , and large $=\mathrm{SMD}>0.8$ [27]. In terms of heterogeneity, we used $\mathrm{I}^{2}$ statistic and evaluated what degree of variance between studies can be attributed to actual differences between the studies rather than to chance [28]. Studies suggested that $\mathrm{I}^{2}$ of $75-100 \%$ indicate considerable heterogeneity, and the heterogeneity threshold was defined as $50 \%$ or more in $\mathrm{I}^{2}$ value and $p<0.10$.

\section{RESULTS}

\section{Study Characteristics}

Initially 804 abstracts were identified with use of Embase, PubMed, Psychinfo, and Web of Science. After a

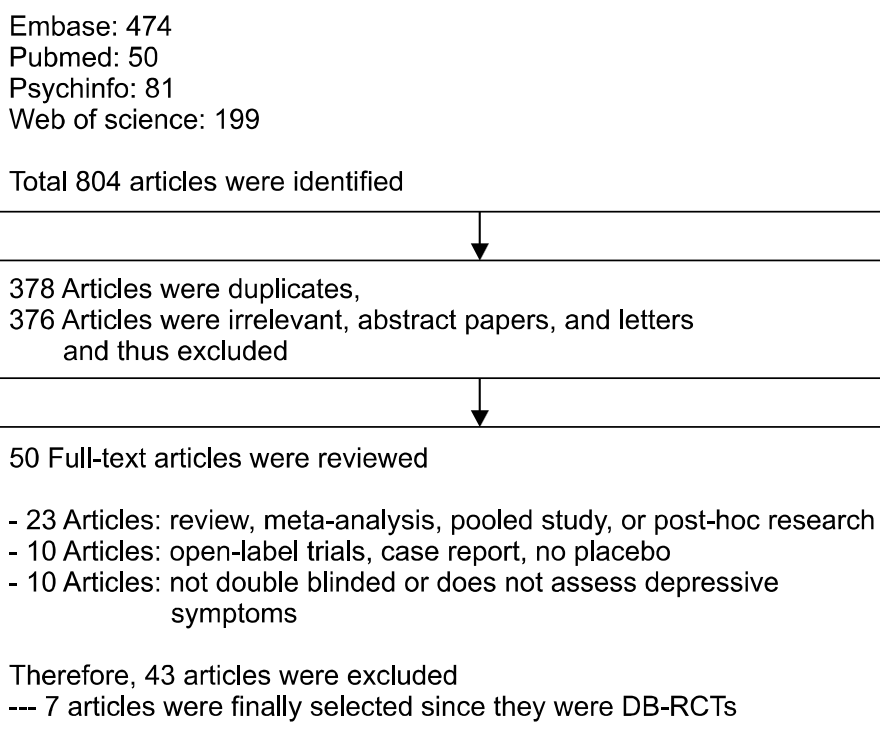

50 Full-text articles were reviewed

- 23 Articles: review, meta-analysis, pooled study, or post-hoc research

- 10 Articles: open-label trials, case report, no placebo

- 10 Articles: not double blinded or does not assess depressive symptoms

Therefore, 43 articles were excluded

--- 7 articles were finally selected since they were DB-RCTs

Total 8 DB-RCTs were selected

Clinicaltrialgov.com: 35

- 25 Clinical trials: results not available

- 1 Clinical trial: relapse prevention study

- 1 Clinical trial: open label study

- 7 Clinical trials: duplications with published studies

- 1 Unpublished DB-RCT was selected

The Cochrane Central Register of Controlled Trials: 132

- 99 Clinical trials: duplications with Pubmed (28) and Embase (71)

- 32 Clinical trials: duplications with Clinicaltrialgov.com

- 25 Clinical trials: found in ICTRP

-- 9 Clinical trials: irrelevant to treatment of depression

-- 7 Clinical trials: duplications with published trials

-- 9 Clinical trials: no results

--- 1 Unpublished was selected for the meta-analysis
Fig. 1. Schematic presentation of studies selected in the present metaanalysis.

DB-RCT, double-blinded, randomized, placebo-controlled clinical trial; ICTRP, international clinical trials registry platform. 


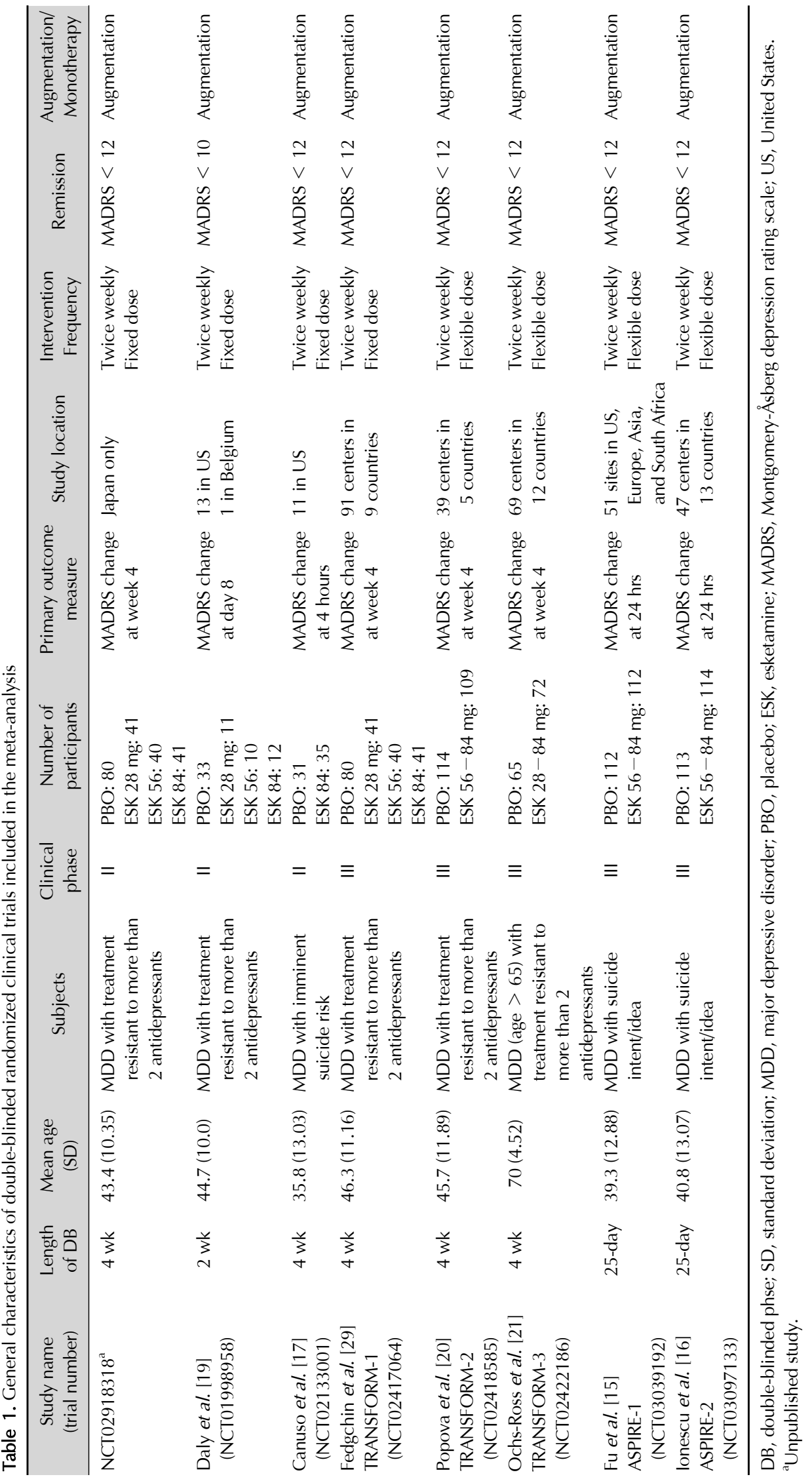


preliminary review, 754 papers were excluded because they were either duplicates, irrelevant, or non-full articles. The remaining 50 full-text articles were retrieved for a more detailed evaluation. Among them seven published DB-RCTs were included in the meta-analysis. Of the 35 records obtained from ClinicalTrials.gov and 132 studies from Cochrane Central Register of Controlled Trials, we found one DB-RCT having full reports which were not published. Thus, a total of eight DB-RCTs (seven published and one un-published) were finally selected for our meta-analysis (Fig. 1).

Table 1 presents main characteristics of these eight DB-RCTs. All studies were multi-centered, and six studies $[15,16,19-21,29]$ were multi-national while two were conducted either in Japan [30] or US [17] only. Five trials [19-21,29,30] involved TRD while other three [15-17] involved patients with MDSI. A total of 1,488 participants were included, and number of patients included in placebo and intranasal esketamine groups were 661 and 827 respectively. Four $[15,16,20,21]$ studies used flexible doses while other four $[17,19,29,30]$ used fixed doses of intranasal esketamine. Risk of bias assessment showed that all studies included were good in quality in terms of their methodologies (Supplementary Fig. 1; available online). Publication bias could not be tested because only one trial was un-published.

\section{Efficacy}

\section{Primary endpoint: mean change of MADRS}

Mean change of MADRS total score from baseline to $2-$ 4 hours, 24 hours, week 1, and week 3-4 are presented as forest plots (Fig. 2). Intranasal esketamine more significantly improved MADRS total scores than placebo for treating MDD starting from $2-4$ hours after the first injection (SMD, $-0.41[95 \% \mathrm{Cl},-0.58$ to -0.25$], p<$ $0.00001)$, and the significant superiority maintained at 24 hours (SMD, -0.36 [95\% Cl, -0.47 to -0.24$], p<$ $0.00001)$, week 1 (SMD, $-0.25[95 \% \mathrm{Cl},-0.36$ to 0.13], $p<0.0001$ ), and end of double-blinded period (week 3-4) (SMD, -0.25 [95\% Cl, -0.35 to -0.14$], p<$ $0.00001)$. Significant heterogeneities were not reported for $2-4$ hours $\left(\mathrm{I}^{2}=0 \%, p=0.40\right), 24$ hours $\left(\mathrm{I}^{2}=42 \%, p=\right.$ $0.13)$, week $1\left(I^{2}=13 \%, p=0.33\right)$, and week $3-4\left(I^{2}=\right.$ $0 \%, p=0.68)$, so we used fixed effect model for all analyses.
We conducted sub-group analysis for patients with TRD and MDSI. In terms of patients with TRD, MADRS improvement was significantly more superior in intranasal esketamine group than in placebo group from $2-4$ hours (SMD, $-0.67[95 \% \mathrm{Cl},-1.16$ to -0.17$], p=$ 0.008 ) to 24 hours (SMD, $-0.48[95 \% \mathrm{Cl},-0.82$ to -0.13$]$, $p=0.007$ ), week 1 (SMD, $-0.27[95 \% \mathrm{Cl},-0.42$ to -0.12$]$, $p=0.0003)$, and week $3-4$ (SMD, $-0.23[95 \% \mathrm{Cl},-0.37$ to -0.10$], p=0.0007)$. However, only one study assessed MADRS at 2-4 hours after the first injection (Fig. 3). Significant heterogeneity was noted for 24 hours $\left(\mathrm{I}^{2}=\right.$ $73 \%, p=0.02$ ), so random effect model was used. For $2-$ 4 hours, week 1 , and week $3-4$ fixed effect model was utilized because no significant heterogeneity was observed. Similar trends of rapid antidepressive effects were noted for subgroup analysis involving patients with MDSI at $2-$ 4 hours (SMD, $-0.38[95 \% \mathrm{Cl},-0.56$ to -0.21$], p<$ $0.0001), 24$ hours (SMD, $-0.34[95 \% \mathrm{Cl},-0.52$ to -0.17$]$, $p=0.0001$ ), week 1 (SMD, -0.21 [95\% Cl, -0.39 to -0.02$]$, $p=0.03)$, and week $3-4$ (SMD, $-0.27[95 \% \mathrm{Cl},-0.44$ to -0.10$], p=0.002$ ) (for all heterogeneity $=0$ ), which is illustrated in Figure 4.

\section{Resolution of suicide}

Esketamine showed superior efficacy over placebo in resolution of suicide at $2-4$ hours after initial nasal infusion with OR of $2.04(95 \% \mathrm{Cls}, 1.37$ to $3.05, p=$ 0.0005 ; heterogeneity $=0 \%$ ), but the two groups did not statistically differ at 24 hours $(\mathrm{OR}=1.15,95 \% \mathrm{Cls}, 0.80$ to $1.65, p=0.46$; heterogeneity $=0 \%)$ and week $3-4(\mathrm{OR}=$ $1.32,95 \%$ Cls, 0.91 to $1.90, p=0.44$; heterogeneity = $0 \%$ ) (Supplementary Fig. 2; available online).

\section{Rate of remission}

A total of seven studies were included for comparing rate of remission between intranasal esketamine and placebo groups at week $3-4$. Intranasal esketamine group showed superior remission rate than placebo with OR of 1.64 (95\% Cls, 1.30 to $2.07, p<0.0001$; heterogeneity = $0 \%$ ) at week $3-4$. In addition, superior efficacy was noted at $2-4$ hours $(\mathrm{OR}=2.43,95 \% \mathrm{Cls}, 1.27$ to $4.67, p=$ 0.007 ; heterogeneity $=41 \%, p=0.18)$ and 24 hours (OR $=$ $2.47,95 \%$ Cls, 1.58 to $3.85, p<0.0001$; heterogeneity $=$ $0 \%$ ) after initial nasal esketamine infusion, but the two groups did not differ at day $8(\mathrm{OR}=1.46,95 \% \mathrm{Cls}, 0.96$ to 2.23, $p=0.08$; heterogeneity $=0 \%$ ) (Supplementary Fig. 
A MADRS change at 2-4 hours Study or subgroup

Canuso et al. [17] Esketamine $84 \mathrm{mg}$

Daly et al. [19] Esketamine 28-84 mg

ASPIRE-1 Fu et al. [15] Esketamine 56-84 mg

ASPIRE-2 lonescu et al. [16] Esketamine 56-84 mg

Total $(95 \% \mathrm{Cl})$

Heterogeneity: $\mathrm{Chi}^{2}=2.97, \mathrm{df}=3(p=0.40) ;\left.\right|^{2}=0 \%$

Test for overall effect: $Z=4.91(p<0.00001)$

B MADRS change at 24 hours

Study or subgroup

Canuso et al. [17] Esketamine $84 \mathrm{mg}$

Daly et al. [19] Esketamine 28-84 mg

TRANSFORM-1 Fedgchin et al. [29] Esketamine $56-84 \mathrm{mg}$

TRANSFORM-2 Popova et al. [20] Esketamine $56-84 \mathrm{mg}$

ASPIRE-1 Fu et al. [15] Esketamine 56-84 mg

ASPIRE-2 lonescu et al. [16] Esketamine 56-84 mg

Total $(95 \% \mathrm{Cl})$

Heterogeneity: $\mathrm{Chi}^{2}=8.55, \mathrm{df}=5(p=0.13) ;\left.\right|^{2}=42 \%$

Test for overall effect: $Z=5.86(p<0.00001)$

C MADRS change at week 1

Study or subgroup

Daly et al. [19] Esketamine 28-84 mg

TRANSFORM-1 Fedgchin et al. [29] Esketamine $56-84 \mathrm{mg}$

TRANSFORM-3 Ochs-Ross et al. [21] Esketamine 28-84 mg

TRANSFORM-2 Popova et al. [20] Esketamine $56-84 \mathrm{mg}$

ASPIRE-1 Fu et al. [15] Esketamine 56-84 mg

ASPIRE-2 lonescu et al. [16] Esketamine 56-84 mg

Total $(95 \% \mathrm{Cl})$

Heterogeneity: $\mathrm{Chi}^{2}=5.78, \mathrm{df}=5(p=0.33) ; I^{2}=13 \%$

Test for overall effect: $Z=4.19(p<0.0001)$

D MADRS change at week 3-4

Study or subgroup

ASPIRE-1 Fu et al. [15] Esketamine 56-84 mg

ASPIRE-2 lonescu et al. [16] Esketamine 56-84 mg

Canuso et al. [17] Esketamine $84 \mathrm{mg}$

NCT02918318

TRANSFORM-1 Fedgchin et al. [29] Esketamine $56-84 \mathrm{mg}$

TRANSFORM-2 Popova et al. [20] Esketamine $56-84 \mathrm{mg}$

TRANSFORM-3 Ochs-Ross et al. [21] Esketamine 28-84 mg

Total $(95 \% \mathrm{Cl})$

Heterogeneity: $\mathrm{Chi}^{2}=3.97, \mathrm{df}=6(p=0.68) ;\left.\right|^{2}=0 \%$

Test for overall effect: $Z=4.55(p<0.00001)$

$\begin{array}{rcr}\text { Weight } & \begin{array}{c}\text { Std. Mean difference } \\ \text { IV, fixed, 95\% Cl }\end{array} & \text { Ye } \\ 11.1 \% & -0.58[-1.07,-0.08] & 2018 \\ 11.1 \% & -0.67[-1.16,-0.17] & 2018 \\ 39.0 \% & -0.25[-0.51,0.01] & 2020 \\ 38.8 \% & -0.45[-0.72,-0.19] & 2020 \\ & & \\ 100.0 \% & -0.41[-0.58,-0.25]\end{array}$

Std. Mean difference
IV, fixed, $95 \% \mathrm{Cl}$

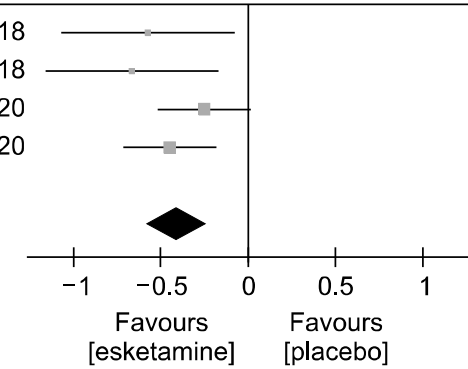

[esketamine] [placebo]

$\begin{array}{rcc}\text { Weight } & \begin{array}{c}\text { Std. Mean difference } \\ \text { IV, fixed, 95\% Cl }\end{array} & \text { Year } \\ 5.8 \% & -0.58[-1.08,-0.09] & 2018 \\ 5.4 \% & -1.02[-1.54,-0.51] & 2018 \\ 27.6 \% & -0.25[-0.47,-0.02] & 2019 \\ 20.2 \% & -0.36[-0.62,-0.09] & 2019 \\ 20.3 \% & -0.32[-0.58,-0.05] & 2020 \\ 20.6 \% & -0.30[-0.56,-0.04] & 2020 \\ & & \\ 100.0 \% & -0.36[-0.47,-0.24] & \end{array}$

Std. Mean difference IV, fixed, $95 \% \mathrm{Cl}$

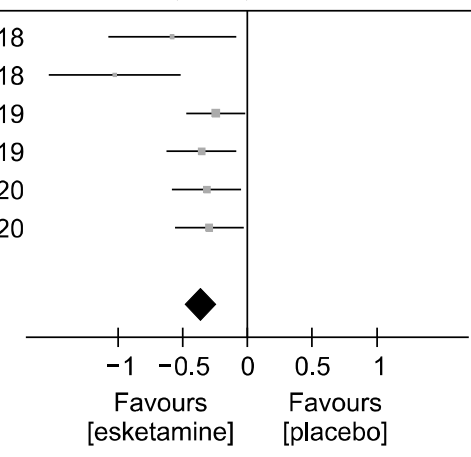

\begin{tabular}{|c|c|c|c|c|c|}
\hline Weight & $\begin{array}{l}\text { Std. Mean difference } \\
\text { IV, fixed, } 95 \% \mathrm{Cl}\end{array}$ & Year & \multicolumn{3}{|c|}{$\begin{array}{l}\text { Std. Mean difference } \\
\text { IV, fixed, } 95 \% \mathrm{Cl}\end{array}$} \\
\hline $5.3 \%$ & $-0.80[-1.30,-0.30]$ & 2018 & - & & \\
\hline $25.9 \%$ & $-0.22[-0.45,0.00]$ & 2019 & & & \\
\hline $11.7 \%$ & $-0.11[-0.44,0.23]$ & 2019 & & - & \\
\hline $18.5 \%$ & $-0.28[-0.55,-0.02]$ & 2019 & & & \\
\hline $19.2 \%$ & $-0.18[-0.44,0.08]$ & 2020 & & & \\
\hline $19.4 \%$ & $-0.24[-0.50,0.02]$ & 2020 & & & \\
\hline \multirow[t]{3}{*}{$100.0 \%$} & $-0.25[-0.36,-0.13]$ & & & & \\
\hline & & & $\begin{array}{ll}-1 & -0.5\end{array}$ & 0.5 & 1 \\
\hline & & & $\begin{array}{c}\text { Favours } \\
\text { [esketamine] }\end{array}$ & $\begin{array}{r}\text { Favours } \\
\text { [placebo] }\end{array}$ & \\
\hline
\end{tabular}

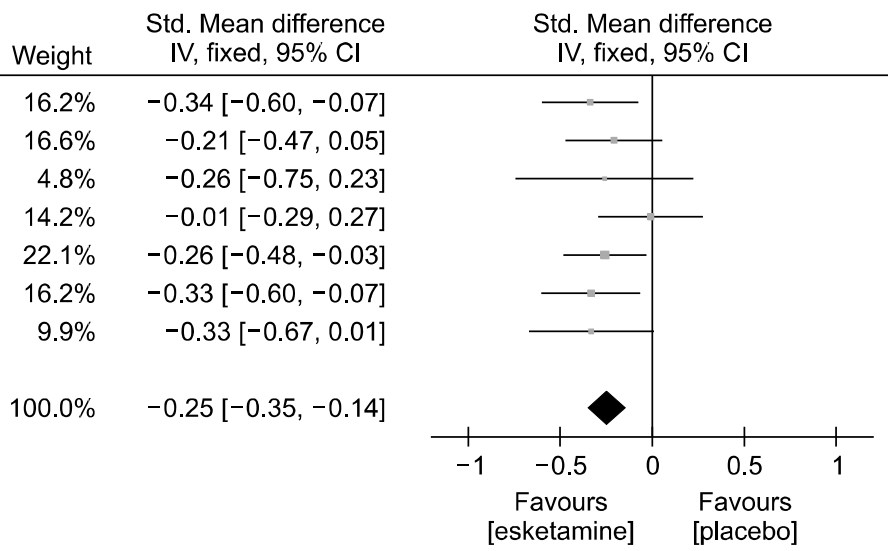

Fig. 2. Mean change of Montgomery-Åsberg depression rating scale (MADRS) at (A) $2-4$ hours, (B) 24 hours, (C) week 1, and (D) week $3-4$ between intranasal esketamine and placebo.

Std., standard deviation; $\mathrm{Cl}$, confidence interval; IV, inverse variance.

a Unpublished study. 


\begin{tabular}{|c|c|c|c|c|c|}
\hline \multirow{2}{*}{$\begin{array}{l}\text { A MADRS change at } 2-4 \text { hours } \\
\text { Study or subgroup } \\
\text { Daly et al. [19] Esketamine } 28-84 \mathrm{mg}\end{array}$} & \multicolumn{3}{|c|}{ Std. Mean difference } & \multicolumn{2}{|c|}{$\begin{array}{l}\text { Std. Mean difference } \\
\text { IV, fixed, } 95 \% \mathrm{Cl}\end{array}$} \\
\hline & $100.0 \%$ & $-0.67[-1.16,-0.17]$ & 2018 & & \\
\hline Total $(95 \% \mathrm{Cl})$ & $100.0 \%$ & $-0.67[-1.16,-0.17]$ & & & \\
\hline \multicolumn{6}{|l|}{ Heterogeneity: Not applicable } \\
\hline \multirow[t]{2}{*}{ Test for overall effect: $Z=2.65(p=0.008)$} & & & & $\begin{array}{lll}-1 & -0.5 & 0\end{array}$ & 0.51 \\
\hline & & & & $\begin{array}{c}\text { Favours } \\
\text { [esketamine] }\end{array}$ & $\begin{array}{l}\text { Favours } \\
\text { [placebo] }\end{array}$ \\
\hline
\end{tabular}

B MADRS change at 24 hours

\section{Study or subgroup}

Daly et al. [19] Esketamine 28-84 mg

TRANSFORM-1 Fedgchin et al. [29] Esketamine $56-84 \mathrm{mg}$

TRNASFORM-2 Popova et al. [20] Esketamine 56-84 mg

\begin{tabular}{cc} 
Weight & $\begin{array}{c}\text { Std. Mean difference } \\
\text { IV, random, 95\% Cl }\end{array}$ \\
\hline $23.3 \%$ & $-1.02[-1.54,-0.51]$ \\
$39.5 \%$ & $-0.25[-0.47,-0.02]$ \\
$37.1 \%$ & $-0.38[-0.64,-0.11]$ \\
& \\
$100.0 \%$ & $-0.48[-0.82,-0.13]$
\end{tabular}

Total $(95 \% \mathrm{Cl})$

Heterogeneity: $\mathrm{Tau}^{2}=0.07, \mathrm{Chi}^{2}=7.44, \mathrm{df}=2(p=0.02) ; \mathrm{I}^{2}=73 \%$

Test for overall effect: $Z=2.70(p=0.007)$
Std. Mean difference $\mathrm{IV}$, random, $95 \% \mathrm{Cl}$

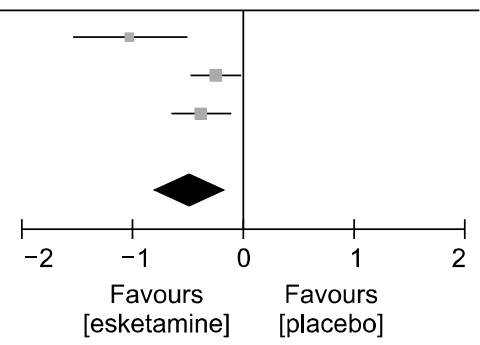

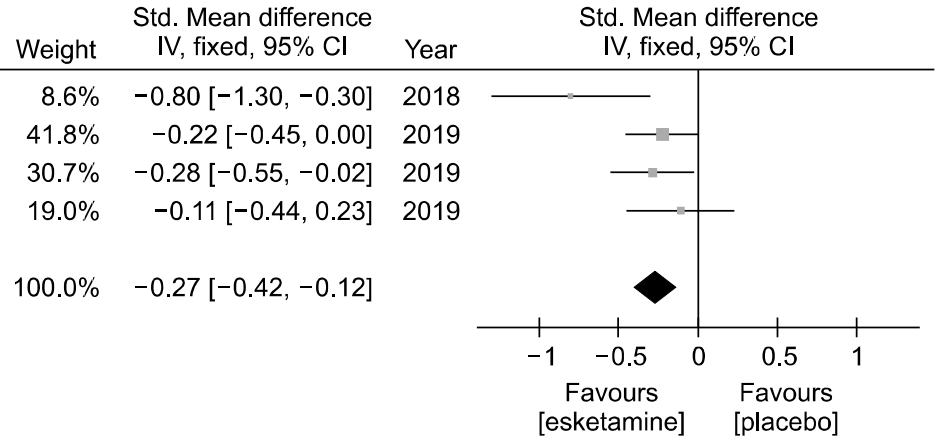
[esketamine] [placebo]

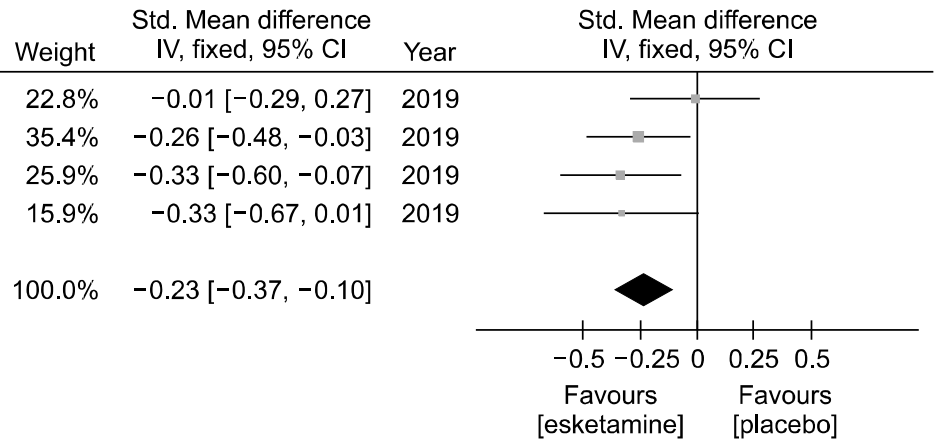

Fig. 3. Mean change of Montgomery-Åsberg depression rating scale (MADRS) at (A) $2-4$ hours, (B) 24 hours, (C) week 1, and (D) week $3-4$ between intranasal esketamine and placebo in patients with treatment resistant depression (TRD).

Std., standard deviation; Cl, confidence interval; IV, inverse variance.

a Unpublished study.

3; available online).

\section{Safety and Tolerability}

In terms of commonly observed side effects, esketamine showed higher incidence of total $\mathrm{AEs}(\mathrm{OR}=4.23$, $95 \% \mathrm{Cls}, 2.85$ to $6.27, p<0.00001$; heterogeneity $=$
$55 \%, p=0.04$ ), dissociation ( $\mathrm{OR}=7.93,95 \% \mathrm{Cls}, 5.36$ to $11.72, p<0.00001$; heterogeneity $=0 \%$ ), blood pressure increment $(\mathrm{OR}=7.18,95 \% \mathrm{Cls}, 4.82$ to $10.69, p<$ 0.00001 ; heterogeneity $=0 \%)$, nausea $(\mathrm{OR}=3.28,95 \%$ Cls, 2.40 to $4.48, p<0.00001$; heterogeneity $=30 \%, p=$ 0.20 ), vertigo ( $\mathrm{OR}=6.22,95 \% \mathrm{Cls}, 3.97$ to $9.73, p<$ 
A MADRS change at 2-4 hours Study or subgroup

Canuso et al. [17] Esketamine $84 \mathrm{mg}$ ASPIRE-1 Fu et al. [15] Esketamine 56-84 mg ASPIRE-2 lonescu et al. [16] Esketamine 56-84 mg

Total $(95 \% \mathrm{Cl})$

Heterogeneity: $\mathrm{Chi}^{2}=1.91, \mathrm{df}=2(p=0.38) ; I^{2}=0 \%$

Test for overall effect: $Z=4.32(p<0.0001)$

\begin{tabular}{|c|c|c|c|c|c|c|c|}
\hline Weight & $\begin{array}{l}\text { Std. Mean difference } \\
\text { IV, fixed, } 95 \% \mathrm{Cl}\end{array}$ & Year & \multicolumn{5}{|c|}{$\begin{array}{l}\text { Std. Mean difference } \\
\text { IV, fixed, } 95 \% \mathrm{Cl}\end{array}$} \\
\hline $13.1 \%$ & $-0.59[-1.06,-0.11]$ & 2018 & & & & & \\
\hline $43.5 \%$ & $-0.25[-0.51,0.01]$ & 2020 & & & & & \\
\hline $43.5 \%$ & $-0.45[-0.72,-0.19]$ & 2020 & & & & & \\
\hline \multirow[t]{3}{*}{$100.0 \%$} & $-0.38[-0.56,-0.21]$ & & & & & & \\
\hline & & & -1 & -0.5 & 0 & 0.5 & 1 \\
\hline & & & & $\begin{array}{l}\text { Favours } \\
\text { lacebo] }\end{array}$ & & $\begin{array}{l}\text { Eavou } \\
\text { ketan }\end{array}$ & \\
\hline
\end{tabular}

B MADRS change at 24 hours Study or subgroup

Canuso et al. [17] Esketamine $84 \mathrm{mg}$

ASPIRE-2 lonescu et al. [16] Esketamine 56-84 mg ASPIRE-1 Fu et al. [15] Esketamine 56-84 mg

Total $(95 \% \mathrm{Cl})$

Heterogeneity: $\mathrm{Chi}^{2}=1.05, \mathrm{df}=2(p=0.59) ; I^{2}=0 \%$

Std. Mean difference

Std. Mean difference

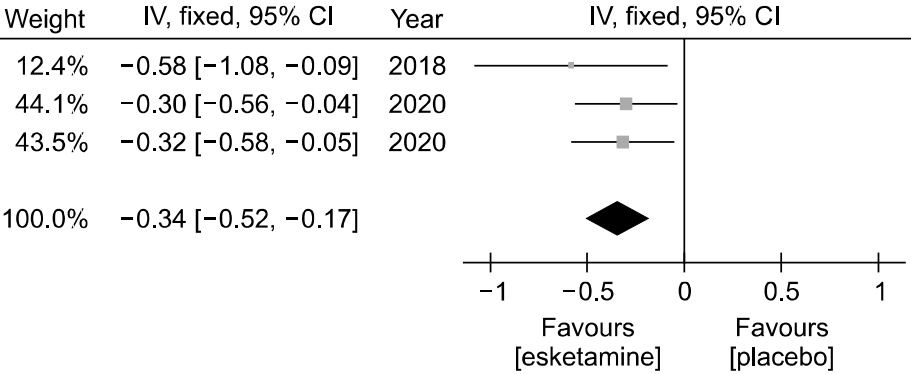

C MADRS change at week 1
Study or subgroup

Std. Mean difference IV, fixed, $95 \% \mathrm{Cl}$

ASPIRE-1 Fu et al. [15] Esketamine 28-84 mg

ASPIRE-2 lonescu et al. [16] Esketamine 56-84 mg

Weight

-0.18[-0.44,0.08]

$\begin{array}{ll}49.7 \% & -0.18[-0.44,0.08] \\ 50.3 \% & -0.24[-0.50,0.02]\end{array}$

Total $(95 \% \mathrm{Cl})$

Heterogeneity: $\mathrm{Chi}^{2}=0.09, \mathrm{df}=1(p=0.76) ; \mathrm{I}^{2}=0 \%$

Test for overall effect: $Z=2.21(p=0.03)$

$100.0 \%-0.21[-0.39,-0.02]$

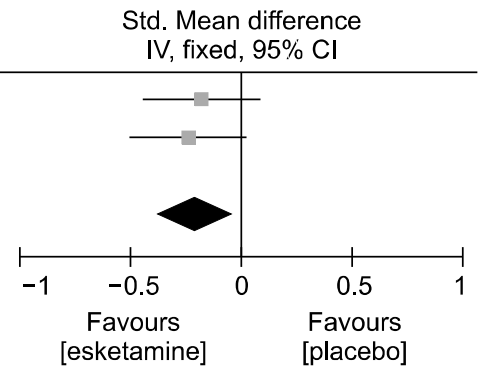

D MADRS change at week 3-4

Study or subgroup

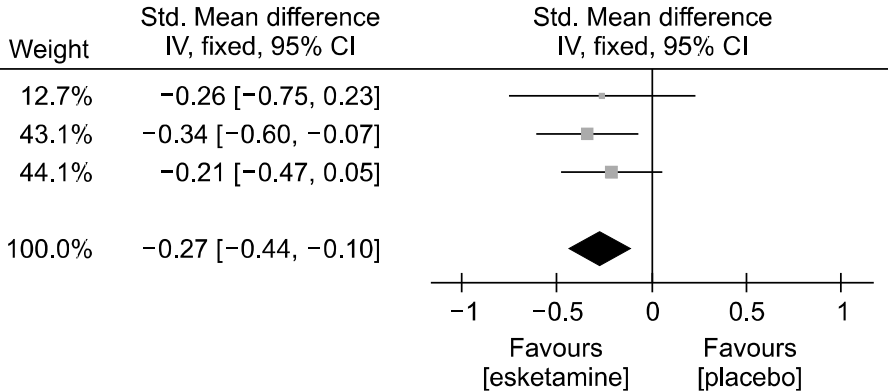

Fig. 4. Mean change of Montgomery-Åsberg depression rating scale (MADRS) at (A) $2-4$ hours, (B) 24 hours, (C) week 1 , and (D) week $3-4$ between intranasal esketamine and placebo in major depression with suicide ideation (MDSI).

Std., standard deviation; $\mathrm{Cl}$, confidence interval; IV, inverse variance.

0.00001 ; heterogeneity $=43 \%, p=0.10)$, dysgeusia $(\mathrm{OR}=$ $1.67,95 \%$ Cls, 1.21 to $2.31, p=0.002$; heterogeneity = $0 \%)$, dizziness $(\mathrm{OR}=4.47,95 \% \mathrm{Cls}, 3.27$ to $6.11, p<$ 0.00001 ; heterogeneity $=0 \%$ ), and somnolence (OR = $2.08,95 \%$ Cls, 1.49 to $2.89, p<0.0001$; heterogeneity = $0 \%$ ) compared with placebo (Fig. 5). Although headache was numerically more common in esketamine group than in placebo group, the two groups did not differ statistically $(\mathrm{OR}=1.33,95 \% \mathrm{Cls}, 1.00$ to $1.77, p=0.05$; heterogeneity $=5 \%, p=0.39$ ) . 


\begin{tabular}{|c|c|c|c|c|c|c|c|c|}
\hline \multirow{2}{*}{$\begin{array}{l}\text { A Total } \\
\text { Study or subgroup } \\
\text { Canuso et al. [17] Esketamine } 84 \mathrm{mg}\end{array}$} & Weight & \multicolumn{2}{|c|}{ Odds ratio (non-event) } & \multicolumn{4}{|c|}{$\begin{array}{l}\text { Odds ratio (non-event) } \\
\mathrm{M}-\mathrm{H} \text {, random, } 95 \% \mathrm{Cl}\end{array}$} & \\
\hline & $4.6 \%$ & $3.96[0.74,21.30]$ & 2018 & & & & & \\
\hline NCT02918318 & $13.5 \%$ & $13.65[6.37,29.24]$ & 2019 & & & & & \\
\hline TRANSFORM-1 Fedgchin et al. [29] Esketamine 56-84 mg & $19.0 \%$ & $3.22[1.96,5.29]$ & 2019 & & & & $\longrightarrow$ & \\
\hline TRANSFORM-2 Popova et al. [20] Esketamine $56-84 \mathrm{mg}$ & $16.3 \%$ & $5.45[2.94,10.10]$ & 2019 & & & & $\longrightarrow$ & \\
\hline TRANSFORM-3 Ochs-Ross et al. [21] Esketamine $28-84 \mathrm{mg}$ & $14.6 \%$ & $3.07[1.53,6.18]$ & 2019 & & & & - - & \\
\hline ASPIRE-1 Fu et al. [15] Esketamine 56-84 mg & $16.5 \%$ & $3.02[1.65,5.53]$ & 2020 & & & & $\longrightarrow$ & \\
\hline ASPIRE-2 lonescu et al. [16] Esketamine 56-84 mg & $15.5 \%$ & $3.23[1.68,6.22]$ & 2020 & & & & - & \\
\hline Total $(95 \% \mathrm{Cl})$ & $100.0 \%$ & $4.23[2.85,6.27]$ & & & & & & \\
\hline \multicolumn{4}{|l|}{ Total events } & 0.05 & 0.2 & 1 & 5 & 20 \\
\hline Test for overall effect: $Z=7.18(p<0.00001)$ & & & & & $\begin{array}{l}\text { Favou } \\
\text { sketan }\end{array}$ & & $\begin{array}{l}\text { Favours } \\
\text { [placebo] }\end{array}$ & \\
\hline \multicolumn{2}{|l|}{$\begin{array}{l}\text { B Dissociation } \\
\text { Study or subgroup }\end{array}$} & \multicolumn{2}{|c|}{$\begin{array}{l}\text { Odds ratio (non-event) } \\
\mathrm{M}-\mathrm{H} \text {, fixed, } 95 \% \mathrm{Cl}\end{array}$} & \multicolumn{5}{|c|}{$\begin{array}{l}\text { Odds ratio (non-event) } \\
\mathrm{M}-\mathrm{H} \text {, fixed, } 95 \% \mathrm{Cl}\end{array}$} \\
\hline ASPIRE-1 Fu et al. [15] Esketamine 56-84 mg & $11.7 \%$ & \multicolumn{2}{|c|}{$11.28[3.84,33.13]$} & & & & & \\
\hline ASPIRE-2 lonescu et al. [16] Esketamine 56-84 mg & $22.9 \%$ & \multicolumn{2}{|c|}{$7.26[3.33,15.82]$} & & & & $\Longrightarrow$ & \\
\hline Canuso et al. [17] Esketamine $84 \mathrm{mg}$ & $12.0 \%$ & \multicolumn{2}{|c|}{$3.09[0.87,11.01]$} & & & & & \\
\hline NCT02918318 & $21.8 \%$ & \multicolumn{2}{|c|}{$6.31[2.68,14.88]$} & & & & - & \\
\hline TRANSFORM-1 Fedgchin et al. [29] Esketamine 56-84 mg & $16.1 \%$ & \multicolumn{2}{|c|}{$10.12[3.58,28.61]$} & & & & & \\
\hline TRANSFORM-2 Popova et al. [20] Esketamine $56-84 \mathrm{mg}$ & $11.7 \%$ & \multicolumn{2}{|c|}{$10.44[3.54,30.83]$} & & & & & \\
\hline TRANSFORM-3 Ochs-Ross et al. [21] Esketamine $28-84 \mathrm{mg}$ & $3.8 \%$ & \multicolumn{2}{|c|}{$9.14[1.13,74.29]$} & & & & & \\
\hline Total $(95 \% \mathrm{Cl})$ & $100.0 \%$ & \multicolumn{2}{|c|}{$7.93[5.36,11.72]$} & & & & & \\
\hline Total events & & & & & 1 & & & \\
\hline Heterogeneity: $\mathrm{Chi}^{2}=3.32, \mathrm{df}=6(p=0.77) ; 1^{2}=0 \%$ & & & & 0.01 & 0.1 & 1 & 10 & 100 \\
\hline Test for overall effect: $Z=10.39(p<0.00001)$ & & & & & $\begin{array}{l}\text { Favou } \\
\text { sketan }\end{array}$ & & $\begin{array}{l}\text { Favours } \\
\text { [placebo] }\end{array}$ & \\
\hline \multicolumn{2}{|l|}{$\begin{array}{l}\text { C Blood pressure increment } \\
\text { Study or subgroup }\end{array}$} & \multicolumn{2}{|c|}{$\begin{array}{l}\text { Odds ratio (non-event) } \\
\mathrm{M}-\mathrm{H} \text {, fixed, } 95 \% \mathrm{Cl}\end{array}$} & \multicolumn{4}{|c|}{$\begin{array}{l}\text { Odds ratio (non-event) } \\
\mathrm{M}-\mathrm{H} \text {, fixed, } 95 \% \mathrm{Cl}\end{array}$} & \\
\hline ASPIRE-1 Fu et al. [15] Esketamine 56-84 mg & $20.8 \%$ & $3.61[1.38,9$ & .42] & & & & $\longrightarrow$ & \\
\hline ASPIRE-2 lonescu et al. [16] Esketamine $56-84 \mathrm{mg}$ & $23.2 \%$ & $7.26[3.33,15$ & 82] & & & & - & \\
\hline NCT02918318 & $23.9 \%$ & $6.25[2.77,14$ & 12] & & & & $\longrightarrow$ & \\
\hline TRANSFORM-1 Fedgchin et al. [29] Esketamine $56-84 \mathrm{mg}$ & $16.3 \%$ & $10.12[3.58,28$ & 61] & & & & & \\
\hline TRANSFORM-2 Popova et al. [20] Esketamine $56-84 \mathrm{mg}$ & $11.9 \%$ & $10.44[3.54,30$ & .83] & & & & & \\
\hline TRANSFORM-3 Ochs-Ross et al. [21] Esketamine $28-84 \mathrm{mg}$ & $3.8 \%$ & $9.14[1.13,74$ & .29] & & & & & \\
\hline Total $(95 \% \mathrm{Cl})$ & $100.0 \%$ & $7.18[4.82,10$ & 69] & & & & & \\
\hline Total events & & & & & & & & \\
\hline Heterogeneity: $\mathrm{Chi}^{2}=3.02, \mathrm{df}=5(p=0.70) ; I^{2}=0 \%$ & & & & 0.01 & 0.1 & 1 & 10 & 100 \\
\hline Test for overall effect: $Z=9.69(p<0.00001)$ & & & & & $\begin{array}{l}\text { Favou } \\
\text { sketan }\end{array}$ & & $\begin{array}{l}\text { Favours } \\
\text { [placebo] }\end{array}$ & \\
\hline
\end{tabular}

Fig. 5. Safety and tolerability: Rate of (A) total, (B) dissociation, (C) blood pressure increment, (D) nausea, (E) vertigo, (F) dysgeusia, (G) dizziness, (H) somnolence, and (I) headache during the double-blind phase.

$\mathrm{Cl}$, confidence interval.

${ }^{a}$ Unpublished study.

\section{DISCUSSION}

To the best of our knowledge, this is the largest metaanalysis (eight DB-RCTs with 1,488 subjects) comparing efficacy and safety of intranasal esketamine and placebo in patients with MDD. Our study confirmed previous research by showing that augmentation of antidepressants with intranasal esketamine was significantly more effective than with placebo for MADRS score change and depression remission $[24,25]$. In addition, the superior treat- 


\begin{tabular}{lr} 
D Nausea & \\
Study or subgroup & Weight \\
\hline ASPIRE-1 Fu et al. [15] Esketamine $56-84 \mathrm{mg}$ & $24.3 \%$ \\
ASPIRE-2 lonescu et al. [16] Esketamine 56-84 mg & $21.8 \%$ \\
Canuso et al. [17] Esketamine $84 \mathrm{mg}$ & $1.4 \%$ \\
NCT02918318 & \\
TRANSFORM-1 Fedgchin et al. [29] Esketamine 56-84 mg & $14.1 \%$ \\
TRANSFORM-2 Popova et al. [20] Esketamine 56-84 mg & $23.0 \%$ \\
TRANSFORM-3 Ochs-Ross et al. [21] Esketamine 28-84 mg & $10.1 \%$
\end{tabular}

Total $(95 \% \mathrm{Cl})$

$100.0 \%$

Heterogeneity: $\mathrm{Chi}^{2}=8.52, \mathrm{df}=6(p=0.20) ; 1^{2}=30 \%$

Test for overall effect: $Z=7.47(p<0.00001)$

\section{E Vertigo \\ Study or subgroup}

ASPIRE-1 Fu et al. [15] Esketamine 56-84 mg

ASPIRE-2 lonescu et al. [16] Esketamine 56-84 mg

Canuso et al. [17] Esketamine $84 \mathrm{mg}$

NCT02918318

TRANSFORM-1 Fedgchin et al. [29] Esketamine 56-84 mg

TRANSFORM-3 Ochs-Ross et al. [21] Esketamine 28-84 mg

Total $(95 \% \mathrm{Cl})$

Total events

Heterogeneity: $\mathrm{Chi}^{2}=10.54, \mathrm{df}=6(p=0.10) ; \mathrm{I}^{2}=43 \%$

Test for overall effect: $Z=8.00(p<0.00001)$
TRANSFORM-2 Popova et al. [20] Esketamine 56-84 mg

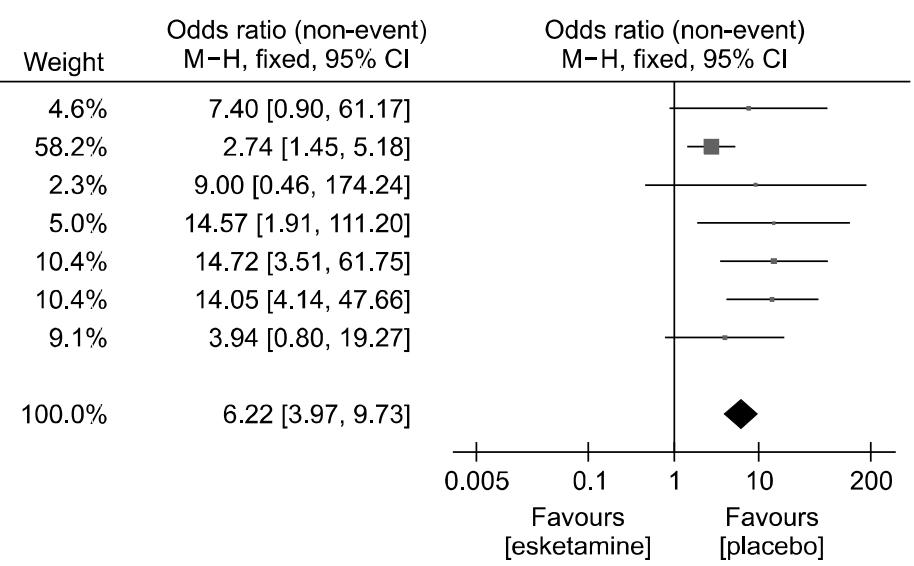

F Dysgeusia

Study or subgroup

ASPIRE-1 Fu et al. [15] Esketamine 56-84 mg

ASPIRE-2 lonescu et al. [16] Esketamine 56-84 mg

Canuso et al. [17] Esketamine $84 \mathrm{mg}$

TRANSFORM-1 Fedgchin et al. [29] Esketamine 56-84 mg

TRANSFORM-2 Popova et al. [20] Esketamine 56-84 mg

TRANSFORM-3 Ochs-Ross et al. [21] Esketamine $28-84 \mathrm{mg}$

Total $(95 \% \mathrm{Cl})$

Total events

Heterogeneity: $\mathrm{Chi}^{2}=4.08, \mathrm{df}=5(p=0.54) ; \mathrm{I}^{2}=0 \%$

Test for overall effect: $Z=3.11(p=0.002)$

Fig. 5. Continued 1.

ment response and remission of intranasal esketamine were noticeable as early as $2-4$ hours after the first intranasal esketamine, and this superior efficacy lasted until end of double blinded phase, which is week 3-4.

By conducting subgroup analysis, we are the first one to show that the rapid improvement of depressive symptoms was evident in patients with TRD and MDSI. Our results also extended previous studies and showed rapid anti-sui-

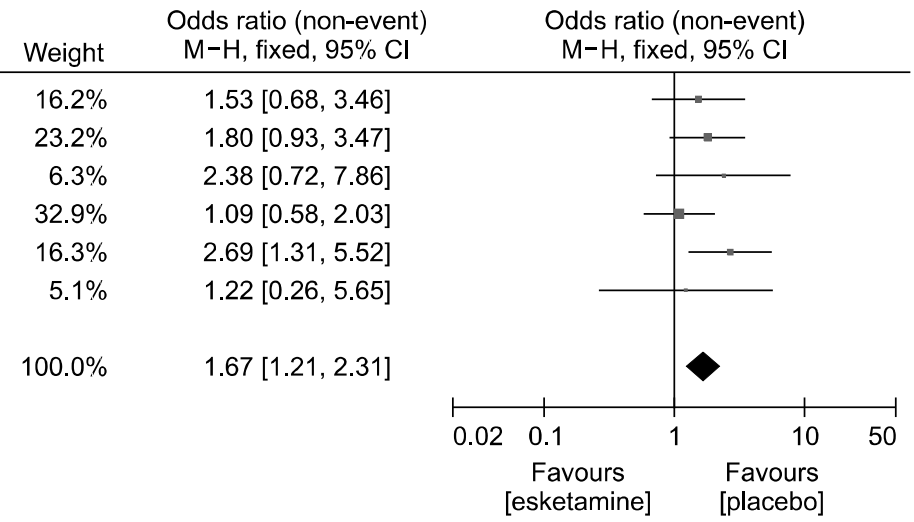

cidal effect of intranasal esketamine (resolution of suicidality 2-4 hours after the 1 st injection) in MDSI. However, although intranasal esketamine showed trend of superior efficacy over placebo, the statistical significance was not maintained at 24 hours and week 3-4. Only three studies were conducted in MDSI, so small number of clinical trials might have been the main cause. More DB-RCTS are needed to define rapid anti-suicidal effects of intra-nasal 


\begin{tabular}{l} 
G Dizziness \\
Study or subgroup \\
\hline ASPIRE-1 Fu et al. [15] Esketamine $56-84 \mathrm{mg}$ \\
ASPIRE-2 lonescu et al. [16] Esketamine $56-84 \mathrm{mg}$ \\
Canuso et al. [17] Esketamine $84 \mathrm{mg}$ \\
NCT02918318 \\
TRANSFORM-1 Fedgchin et al. [29] Esketamine 56-84 \\
TRANSFORM-2 Popova et al. [20] Esketamine 56-8 \\
TRANSFORM-3 Ochs-Ross et al. [21] Esketamine 28 \\
Total (95\% Cl) \\
Total events \\
Heterogeneity: Chi ${ }^{2}=5.79$, df $=6(p=0.45) ; I^{2}=0 \%$ \\
Test for overall effect: $Z=9.36(p<0.00001)$
\end{tabular}

\begin{tabular}{|c|c|c|c|c|c|}
\hline Weight & $\begin{array}{l}\text { Odds ratio (non-event) } \\
\mathrm{M}-\mathrm{H} \text {, fixed, } 95 \% \mathrm{Cl}\end{array}$ & \multicolumn{4}{|c|}{$\begin{array}{l}\text { Odds ratio (non-event) } \\
\mathrm{M}-\mathrm{H} \text {, fixed, } 95 \% \mathrm{Cl}\end{array}$} \\
\hline $14.9 \%$ & $5.67[2.66,12.07]$ & & & - & \\
\hline $28.5 \%$ & $3.12[1.71,5.71]$ & & & & \\
\hline $6.5 \%$ & $3.52[1.00,12.43]$ & & & & \\
\hline $8.4 \%$ & $10.07[3.80,26.69]$ & & & & \\
\hline $23.2 \%$ & $3.49[1.71,7.14]$ & & & & \\
\hline $8.9 \%$ & $6.16[2.25,16.81]$ & & & & \\
\hline $9.7 \%$ & $3.16[1.08,9.25]$ & & & - & \\
\hline \multirow[t]{3}{*}{$100.0 \%$} & $4.47[3.27,6.11]$ & & & & \\
\hline & & 0.02 & 0.1 & 10 & 50 \\
\hline & & & $\begin{array}{l}\text { Favours } \\
\text { esketamine] }\end{array}$ & $\begin{array}{l}\text { Favours } \\
\text { [placebo] }\end{array}$ & \\
\hline
\end{tabular}

\begin{tabular}{|c|c|c|c|c|c|c|c|c|c|c|}
\hline $\begin{array}{l}\text { H Somnolence } \\
\text { Study or subgroup }\end{array}$ & $\begin{array}{l}\text { Plac } \\
\text { Events }\end{array}$ & $\begin{array}{l}\text { bo } \\
\text { Total }\end{array}$ & $\begin{array}{l}\text { Esketa } \\
\text { Events }\end{array}$ & $\begin{array}{l}\text { nine } \\
\text { Total }\end{array}$ & Weight & $\begin{array}{l}\text { Odds ratio (non-event) } \\
\mathrm{M}-\mathrm{H} \text {, fixed, } 95 \% \mathrm{Cl}\end{array}$ & & $\begin{array}{l}\text { Odds rat } \\
\mathrm{M}-\mathrm{H}, \mathrm{fi}\end{array}$ & $\begin{array}{l}\text { (non-event) } \\
\text { ed, } 95 \% \mathrm{Cl}\end{array}$ & \\
\hline ASPIRE-1 Fu et al. [15] Esketamine 56-84 mg & 11 & 112 & 21 & 112 & $17.1 \%$ & $2.12[0.97,4.63]$ & & & & \\
\hline ASPIRE-2 lonescu et al. [16] Esketamine $56-84 \mathrm{mg}$ & 12 & 113 & 26 & 114 & $17.8 \%$ & $2.49[1.18,5.22]$ & & & & \\
\hline Canuso et al. [17] Esketamine $84 \mathrm{mg}$ & 2 & 31 & 4 & 35 & $3.6 \%$ & $1.87[0.32,11.00]$ & & & & \\
\hline NCT02918318 & 14 & 80 & 34 & 122 & $23.4 \%$ & $1.82[0.90,3.67]$ & & & & \\
\hline TRANSFORM-1 Fedgchin et al. [29] Esketamine $56-84 \mathrm{mg}$ & 13 & 113 & 45 & 229 & $26.8 \%$ & $1.88[0.97,3.65]$ & & & $\longrightarrow$ & \\
\hline TRANSFORM-2 Popova et al. [20] Esketamine $56-84 \mathrm{mg}$ & 7 & 114 & 15 & 109 & $11.3 \%$ & $2.44[0.95,6.24]$ & & & & \\
\hline Total $(95 \% \mathrm{Cl})$ & & 563 & & 721 & $100.0 \%$ & $2.08[1.49,2.89]$ & & & & \\
\hline Total events & 59 & & 145 & & & & & & & \\
\hline Heterogeneity: $\mathrm{Chi}^{2}=0.58, \mathrm{df}=5(p=0.99) ; I^{2}=0 \%$ & & & & & & & $\longmapsto$ & + & + & -1 \\
\hline Test for overall effect: $Z=4.35(p<0.0001)$ & & & & & & & 0.01 & 0.1 & 10 & 100 \\
\hline & & & & & & & & $\begin{array}{l}\text { Favours } \\
\text { [esketamine] }\end{array}$ & $\begin{array}{l}\text { Favours } \\
\text { [placebo] }\end{array}$ & \\
\hline
\end{tabular}

I Headache
Study or subgroup
ASPIRE-1 Fu et al. [15] Esketamine $56-84 \mathrm{mg}$
ASPIRE-2 lonescu et al. [16] Esketamine $56-84 \mathrm{mg}$
Canuso et al. [17] Esketamine $84 \mathrm{mg}$
NCT02918318
TRANSFORM-1 Fedgchin et al. [29] Esketamine 56-
TRANSFORM-2 Popova et al. [20] Esketamine 56-84
TRANSFORM-3 Ochs-Ross et al. [21] Esketamine 28
Total (95\% CI)
Total events
Heterogeneity: Chi ${ }^{2}=6.28, \mathrm{df}=6(p=0.39) ; I^{2}=5 \%$
Test for overall effect: $\mathrm{Z}=1.93(p=0.05)$

Fig. 5. Continued 2.

esketamine.

The SMD in MADRS across different time ranged from $0.25-0.41$, which equal to small medium effect size according to Cohen's classification [31]. More importantly, all efficacies including MADRS score change, remission of depression, and resolution of suicidality were greatest either at 2-4 hours or 24 hours after the 1 st administration of intranasal esketamine. All patients in the eight

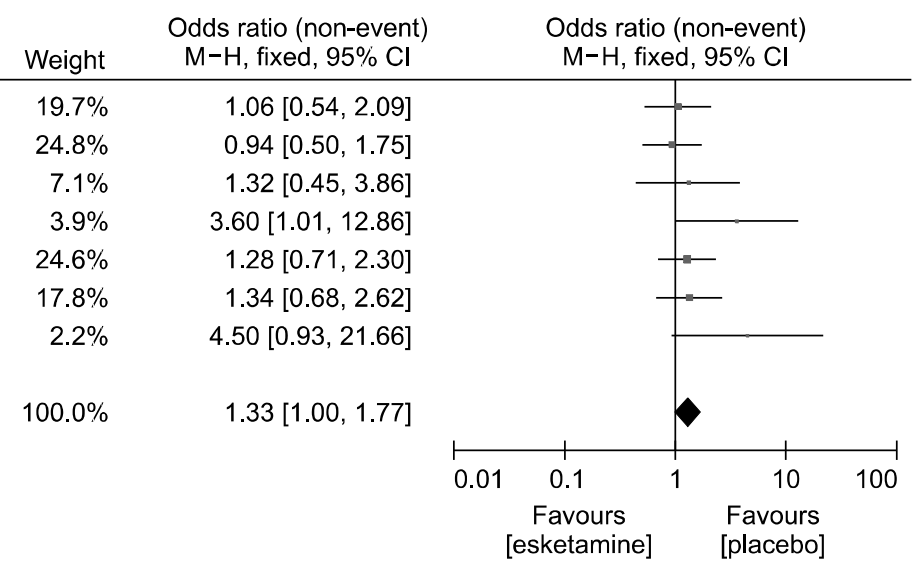

DB-RCTS were taking oral antidepressants in addition to intranasal esketamine or placebo, so the efficacy difference between the two groups might have decreased or attenuated as the onset of actions for oral antidepressants started to show effects. In line with our hypothesis, the mean change of MADRS from baseline to week 3-4 was less than four points difference in all eight DB-RCTs. Thus, as Canuso et al. has suggested, intranasal esketamine 
could be used to overcome the efficacy gap observed between drug administration and onset of action for the conventional antidepressants [17]. This therapeutic role could be particularly important in patients having MDSI.

In terms of safety and tolerability, intranasal esketamine showed higher total AEs than the placebo group. Since esketamine was initially introduced medically as an anesthetic in Germany in 1997 [32], its higher risk of causing somnolence, dizziness, and vertigo are not surprising. The intranasal esketamine also had significantly higher rate of nausea and dysgeusia.

The risk of intranasal esketamine causing dissociation [33] and blood pressure increment [34] have been well documented. Likewise, the rate of dissociation and blood pressure increment was higher in intranasal esketamine than in placebo with particularly higher odd ratios (7.187.93) compared with AEs. Previous studies showed that dissociation and perceptual change symptoms peaked shortly after esketamine administration, which generally resolved by 2 hours after dosing [21,29]. Evidence also showed that rate and intensity of dissociation lowered with repeated administrations of intranasal esketamine. Similarly, studies consistently illustrated that blood pressure increment following intranasal esketamine were transient, asymptomatic, and not associated with serious cardiovascular complications [34]. However, number of studies investing long-term safety and tolerability of intranasal esketamine are scarce [35]. Therefore, whether or not intranasal esketamine result in long-terms adverse events needs further varication.

Our study contained several limitations. First, we combined all doses of intranasal esketamine $(28-84 \mathrm{mg} /$ day $)$ so were not able to conduct meta-regression and investigate its dose related efficacy, tolerability, and safety. Second, we found one unpublished DB-RCT whish showed negative results. There could have been more unpublished negative trials and possibility of publication bias. Third, we did not investigate rate and severity of diverse side effects across different time. As a result, we were not able to confirm that important side effects such as dissociation and blood pressure increment resolved shortly after and attenuated as the administration of intranasal esketamine repeated.

In conclusion, the present meta-analysis confirmed that intranasal esketamine was effective in patients with MDD including TRD and MDSI. Our meta-analysis further showed that intranasal esketamine was associated with rapid antidepressant effect for patients with TRD and MDSI. The study also suggested that esketamine might have rapid anti-suicidal effects for patients with MDSI.

\section{Acknowledgments}

This work was supported by the National Research Foundation of Korea (NRF) grant funded by the Korea government (MSIT) (No. NRF-2019R1C1C1011664).

\section{- Conflicts of Interest}

No potential conflict of interest relevant to this article was reported.

\section{Author Contributions}

Conceptualization: Sheng-Min Wang, Won-Myong Bahk. Data acquisition: Nak-Young Kim, Hae-Ran Na. data analysis: Chi-Un Pae, Hyun Kook Lim, Young Sup Woo. Writing article: Sheng-Min Wang. All authors reviewed and approved for publication.

\section{ORCID}

Sheng-Min Wang https://orcid.org/0000-0003-2521-1413 Nak-Young Kim https://orcid.org/0000-0003-0116-6283 Hae-Ran Na https://orcid.org/0000-0002-7960-8603 Hyun Kook Lim https://orcid.org/0000-0001-8742-3409 Young Sup Woo https://orcid.org/0000-0002-0961-838X Chi-Un Pae https://orcid.org/0000-0003-1632-4248 Won-Myong Bahk https://orcid.org/0000-0002-0156-2510

\section{REFERENCES}

1. Kessler RC, Petukhova M, Sampson NA, Zaslavsky AM, Wittchen $\mathrm{H}-\cup$. Twelve-month and lifetime prevalence and lifetime morbid risk of anxiety and mood disorders in the United States. Int J Methods Psychiatr Res 2012;21:169-184.

2. Fabbri C, Serretti A. Genetics of treatment outcomes in major depressive disorder: present and future. Clin Psychopharmacol Neurosci 2020;18:1-9.

3. Fava M. Diagnosis and definition of treatment-resistant depression. Biol Psychiatry 2003;53:649-659.

4. Rush AJ, Trivedi MH, Wisniewski SR, Nierenberg AA, Stewart JW, Warden D, et al. Acute and longer-term outcomes in depressed outpatients requiring one or several treatment steps: a STAR*D report. Am J Psychiatry 2006;163:1905-1917.

5. Fabbri C, Serretti A. How to utilize clinical and genetic information for personalized treatment of major depressive disorder: step by step strategic approach. Clin Psychopharmacol Neurosci 2020;18:484-492. 
6. Blair-West GW, Cantor CH, Mellsop GW, Eyeson-Annan ML. Lifetime suicide risk in major depression: sex and age determinants. J Affect Disord 1999;55:171-178.

7. Hasin DS, Sarvet AL, Meyers JL, Saha TD, Ruan WJ, Stohl M, et al. Epidemiology of adult DSM-5 major depressive disorder and its specifiers in the United States. JAMA Psychiatry 2018; 75:336-346.

8. Leon AC, Solomon DA, Li C, Fiedorowicz JG, Coryell WH, Endicott J, et al. Antidepressants and risks of suicide and suicide attempts: a 27-year observational study. J Clin Psychiatry 2011;72:580-586.

9. O'Leary OF, Dinan TG, Cryan JF. Faster, better, stronger: towards new antidepressant therapeutic strategies. Eur J Pharmacol 2015; 753:32-50.

10. Wang SM, Han C, Lee SJ, Patkar AA, Masand PS, Pae CU. Five potential therapeutic agents as antidepressants: a brief review and future directions. Expert Rev Neurother 2015;15:10151029.

11. Wang SM, Han C, Pae CU. Criticisms of drugs in early development for the treatment of depression: what can be improved? Expert Opin Investig Drugs 2015;24:445-453.

12. Zanos $P$, Gould TD. Mechanisms of ketamine action as an antidepressant. Mol Psychiatry 2018;23:801-811.

13. Molero P, Ramos-Quiroga JA, Martin-Santos R, Calvo-Sánchez E, Gutiérrez-Rojas L, Meana JJ. Antidepressant efficacy and tolerability of ketamine and esketamine: a critical review. CNS Drugs 2018;32:411-420.

14. Kim J, Farchione T, Potter A, Chen Q, Temple R. Esketamine for treatment-resistant depression - first FDA-approved antidepressant in a new class. N Engl J Med 2019;381:1-4.

15. Fu DJ, lonescu DF, Li X, Lane R, Lim P, Sanacora G, et al. Esketamine nasal spray for rapid reduction of major depressive disorder symptoms in patients who have active suicidal ideation with intent: double-blind, randomized study (ASPIRE I). J Clin Psychiatry 2020;81:19m13191.

16. Ionescu DF, Fu DJ, Qiu X, Lane R, Lim P, Kasper S, et al. Esketamine nasal spray for rapid reduction of depressive symptoms in patients with major depressive disorder who have active suicide ideation with intent: results of a phase 3, double-blind, randomized study (ASPIRE II). Int I Neuropsychopharmacol 2021;24:22-31.

17. Canuso CM, Singh JB, Fedgchin M, Alphs L, Lane R, Lim P, et al. Efficacy and safety of intranasal esketamine for the rapid reduction of symptoms of depression and suicidality in patients at imminent risk for suicide: results of a double-blind, randomized, placebo-controlled study. Am J Psychiatry 2018;175:620-630.

18. FDA approves a nasal spray to treat patients who are suicidal [Internet]. NPR-National Public Radio; 2020 Aug 4 [cited at 2021 Jan 10]. Available from: https://www.npr.org/2020/08/ 04/899060885/fda-approves-a-nasal-spray-to-treat-patientswho-are-suicidal.

19. Daly EJ, Singh JB, Fedgchin M, Cooper K, Lim P, Shelton RC, et al. Efficacy and safety of intranasal esketamine adjunctive to oral antidepressant therapy in treatment-resistant depression: a randomized clinical trial. JAMA Psychiatry 2018;75: 139-148.

20. Popova V, Daly EJ, Trivedi M, Cooper K, Lane R, Lim P, et al. Efficacy and safety of flexibly dosed esketamine nasal spray combined with a newly initiated oral antidepressant in treatment-resistant depression: a randomized double-blind active-controlled study. Am J Psychiatry 2019;176:428-438.

21. Ochs-Ross R, Daly EJ, Zhang Y, Lane R, Lim P, Morrison RL, et al. Efficacy and safety of esketamine nasal spray plus an oral antidepressant in elderly patients with treatment-resistant depression-TRANSFORM-3. Am J Geriatr Psychiatry 2020;28: 121-141.

22. Wang SM, Woo YS, Kim NY, Na HR, Lim HK, Bahk WM. Agomelatine for the treatment of generalized anxiety disorder: a meta-analysis. Clin Psychopharmacol Neurosci 2020;18:423-433.

23. Lyman $\mathrm{GH}$, Kuderer NM. The strengths and limitations of meta-analyses based on aggregate data. BMCMed Res Methodol 2005;5:14.

24. Zheng W, Sim K, Ning YP, Xiang YT. Adjunctive intranasal esketamine for major depressive disorder: a systematic review of randomized double-blind controlled-placebo studies- authors' reply. J Affect Disord 2020;274:955.

25. Papakostas GI, Salloum NC, Hock RS, Jha MK, Murrough JW, Mathew SJ, et al. Efficacy of esketamine augmentation in major depressive disorder: a meta-analysis. J Clin Psychiatry 2020;81:19r12889.

26. Higgins JP, Altman DG, Gøtzsche PC, Jüni $\mathrm{P}$, Moher D, Oxman $\mathrm{AD}$, et al. The Cochrane Collaboration's tool for assessing risk of bias in randomised trials. BMJ 2011;343:d5928.

27. Nakagawa S, Noble DW, Senior AM, Lagisz M. Meta-evaluation of meta-analysis: ten appraisal questions for biologists. BMC Biol 2017;15:18.

28. Melsen WG, Bootsma MC, Rovers MM, Bonten MJ. The effects of clinical and statistical heterogeneity on the predictive values of results from meta-analyses. Clin Microbiol Infect 2014;20:123-129.

29. Fedgchin M, Trivedi M, Daly EJ, Melkote R, Lane R, Lim P, et al. Efficacy and safety of fixed-dose esketamine nasal spray combined with a new oral antidepressant in treatment-resistant depression: results of a randomized, double-blind, activecontrolled study (TRANSFORM-1). Int J Neuropsychopharmacol 2019;22:616-630.

30. A study to evaluate the efficacy, safety and tolerability of fixed doses of intranasal esketamine in Japanese participants with treatment resistant depression [Internet]. U.S. National Library of Medicine; 2019 Dec 13 [cited at 2021 Jan 2]. Available from: https://clinicaltrials.gov/ct2/show/NCTO2918318.

31. Faraone SV. Interpreting estimates of treatment effects: implications for managed care. P T 2008;33:700-711.

32. Himmelseher S, Pfenninger $\mathrm{E}$. [The clinical use of $S-(+)-k e t-$ 
amine--a determination of its place]. Anasthesiol Intensivmed Notfallmed Schmerzther 1998;33:764-770. German.

33. Pereira S, Brennan E, Patel A, Moran M, Wallier J, Liebowitz MR. Managing dissociative symptoms following the use of esketamine nasal spray: a case report. Int Clin Psychopharmacol 2021;36:54-57.

34. Doherty T, Wajs E, Melkote R, Miller J, Singh JB, Weber MA. Cardiac safety of esketamine nasal spray in treatment-resistant depression: results from the clinical development program. CNS Drugs 2020;34:299-310.

35. Wajs E, Aluisio L, Holder R, Daly EJ, Lane R, Lim P, et al. Esketamine nasal spray plus oral antidepressant in patients with treatment-resistant depression: assessment of long-term safety in a phase 3, open-label study (SUSTAIN-2). I Clin Psychiatry 2020;81:19m12891. 